BI-TP 2002/21

\title{
Deep inelastic scattering and "elastic" diffraction
}

\author{
Masaaki Kuroda \\ Institute of Physics, Meiji-Gakuin University \\ Yokohama 244, Japan \\ and \\ Dieter Schildknecht \\ Fakultät für Physik, Universität Bielefeld \\ D-33501 Bielefeld, Germany
}

\begin{abstract}
We examine the total cross section of virtual photons on protons, $\sigma_{\gamma^{*} p}\left(W^{2}, Q^{2}\right)$, at low $x \cong Q^{2} / W^{2} \ll 1$ and its connection with "elastic" diffractive production $\gamma_{T, L}^{*} p \rightarrow X_{T, L}^{J=1} p$ in the two-gluon exchange dynamics for the virtual forward Compton scattering amplitude. Solely based on the generic structure of twogluon exchange, we establish that the cross section is described by the (imaginary part of the) amplitude for forward scattering of $q \bar{q}$ vector states, $(q \bar{q})_{T, L}^{J=1} p \rightarrow$ $(q \bar{q})_{T, L}^{J=1} p$. The generalized vector dominance/color dipole picture (GVD/CDP) is accordingly established to only rest on the two-gluon-exchange generic structure. This is explicitly seen by the sum rules that allow one to directly relate the total cross section to the cross section for elastic diffractive forward production, $\gamma_{T, L}^{*} p \rightarrow(q \bar{q})_{T, L}^{J=1} p$, of vector states.
\end{abstract}




\section{Introduction}

A widely accepted picture of deep inelastic electron scattering on nucleons at low values of $x \cong Q^{2} / W^{2} \ll 1$, in terms of the virtual forward Compton amplitude, is based on the two-gluon exchange dynamical mechanism [1] depicted in fig. 1. The two-gluon exchange mechanism was evaluated 22 in momentum space and its representation [2 in transverse position space 3] became known as the colordipole approach [2]: taking into account the low- $x$ kinematics, the photon in fig. 1 virtually dissociates into a $q \bar{q}$ color dipole that subsequently undergoes diffractive forward scattering on the proton.

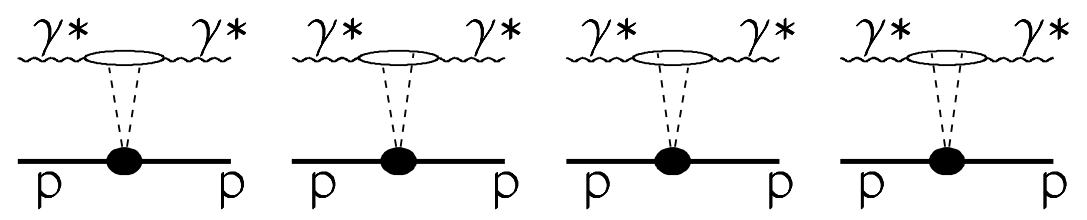

Figure 1: The forward Compton amplitude.

In the present work, we examine the question of which spins of the $q \bar{q}$ system contribute to the forward scattering process $(q \bar{q}) p \rightarrow(q \bar{q}) p$ in the Compton amplitude. It comes without surprise that we find that the process exclusively proceeds via the forward scattering of $J=1$ (i.e. vector) $q \bar{q}$ states, $(q \bar{q})^{J=1}$. Our result is only based on the generic structure of the two-gluon exchange in fig. 1. Obviously, $J=1$ is a consequence of the $\gamma^{*} q \bar{q}$ transition that only allows for interactions of $J=1$ states, $(q \bar{q})^{J=1} p \rightarrow(q \bar{q})^{J=1} p$. The relevance of only $(q \bar{q})^{J=1}$ states is most transparently expressed in terms of a sum rule that relates the total virtual photoabsorption cross section to diffractive forward production (compare fig. 2) of vector states, $\gamma_{T, L}^{*} p \rightarrow(q \bar{q})_{T, L}^{J=1} p$. The sum rule to be given in the present paper coincides with the one obtained in ref. 4] under more restrictive assumptions 
[5] on the $q \bar{q}$ interaction with the proton corresponding to the lower vertices in fig. 1. In the present work, no specific ansatz for the gluon-gluon- $p p$ interaction is introduced, and, in addition, the structure of the previously adopted ansatz [5] is recognized as a realization, without much loss of generality, of the underlying $(q \bar{q})^{J=1} p \rightarrow(q \bar{q})^{J=1} p$ interaction.

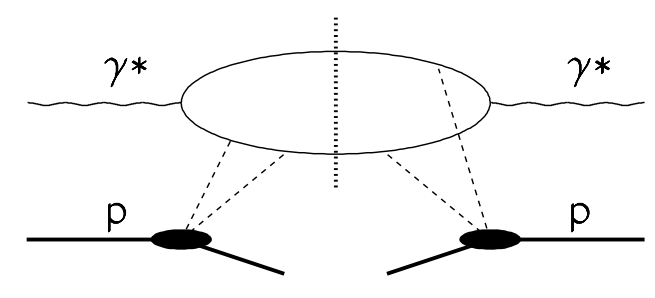

Figure 2: One of the 16 diagrams for diffractive production. The vertical line indicates the unitarity cut corresponding to the diffractively produced final states, $(q \bar{q})^{J}$. Production of (discrete or continuum) vector states corresponds to $(q \bar{q})^{J}$ production with $J=1$.

The emerging picture of deep inelastic scattering at low $x$ coincides with the one of generalized vector dominance (GVD) [6, 7] ${ }^{1}$ from the pre-QCD era; generalized vector dominance is obtained as a consequence of the two-gluon exchange generic structure from QCD. As previously stressed [9], it is precisely the gauge-theory structure underlying the two-gluon interaction with its change in sign between the different contributions in fig. 1 that is responsible for the consistency of GVD. This structure, in terms of cancellations between diagonal and off-diagonal transitions, was anticipated [9, 10] by off-diagonal GVD [7.

The present work clarifies the connection between the total cross section and diffractive production as observed [11] at HERA: the total cross section, $\sigma_{\gamma_{T, L}^{*}}\left(W^{2}, Q^{2}\right)$, is quantitatively related to the amplitudes of "elastic" diffractive production, $\gamma_{T, L}^{*} p \rightarrow(q \bar{q})_{T, L}^{J=1} p$, via the above-mentioned sum rule. "Inelastic" diffraction, $\gamma_{T, L}^{*} p \rightarrow(q \bar{q})^{J \neq 1} p$, (corresponding to diffraction dissociation in

\footnotetext{
${ }^{1}$ Compare also the review on GVD in 8
} 
hadron-hadron interactions) constitutes an additional important contribution to diffractive production, in particular for high invariant masses of the states produced. Inelastic diffraction is irrelevant, however, with respect to the total cross section. It is (obviously) only the elastic component of diffractive production that enters the forward Compton amplitude.

Fits to the experimental data for $\sigma_{\gamma^{*} p}\left(W^{2}, Q^{2}\right)$ or, equivalently $F_{2}\left(x, Q^{2}\right)$, were frequently based [12, 13, 14, on an ansatz for the color-dipole cross section in transverse position space that did not incorporate the restriction to $(q \bar{q})^{J=1}$ states. Since only $(q \bar{q})^{J=1}$ states contribute to the scattering, while all others, $(q \bar{q})^{J \neq 1}$, yield vanishing contributions, the latter ones implicitly remained undetermined in the fits to $\sigma_{\gamma^{*} p}\left(W^{2}, Q^{2}\right)$. This lack of restrictions on the amplitudes for $(q \bar{q})^{J=1} p \rightarrow(q \bar{q})^{J \neq 1} p$ is presumably the reason for widely differing results [15] on the dipole cross section that were extracted from the fits. Dropping the redundant $J \neq 1$ terms in fits to the total cross section, $\sigma_{\gamma_{T, L}^{*}}\left(W^{2}, Q^{2}\right)$, right from the beginning, most likely will improve the uniqueness of the extracted dipole cross sections. We note that the ansatz of the generalized vector dominance/color dipole picture (GVD/CDP) [5] incorporates the restriction to $(q \bar{q})^{J=1}$ states.

In section 2, we use the conventional formalism of the color-dipole model in transverse position space, in order to show that $\sigma_{\gamma^{*} p}\left(W^{2}, Q^{2}\right)$ is determined by the $(q \bar{q})^{J=1} p \rightarrow(q \bar{q})^{J=1} p$ forward scattering amplitude, i.e. by the color-dipole cross section for $J=1$ color-dipoles originating from the $\gamma^{*} \rightarrow q \bar{q}$ dissociation. We show how the redundant amplitudes from scattering into $(q \bar{q})^{J \neq 1}$ states can be eliminated right at the beginning by a slight refinement in the representation of the total cross section in transverse position space.

In section 3, we use the momentum-space representation in order to derive the sum rules that determine the longitudinal and transverse photoabsorption cross sections, $\sigma_{\gamma_{T, L}^{*} p}\left(W^{2}, Q^{2}\right)$, as integrals over the mass spectra of the diffractively produced vector states.

In section 4, we elaborate on the connection between the dipole cross section and the gluon structure function.

In section 5, we remind the reader of the ansatz [5] for the color-dipole cross 
section used in the fits to the total cross section in the GVD/CDP.

In section 6 , we stress the duality relation between the description of low- $x$ deep inelastic scattering in terms of scattering of $(q \bar{q})$ vector states, $(q \bar{q})^{J=1} p \rightarrow$ $(q \bar{q})^{J=1} p$, in the GVD/CDP, and a description in terms of $\gamma^{*}$ gluon scattering, $\gamma^{*} g \rightarrow \gamma^{*} g$ employing the notion of the gluon structure function. The gluon structure function of the GVD/CDP multiplied by $\alpha_{s}\left(Q^{2}\right)$ becomes a function of a single variable $1 / W^{2}=x / Q^{2}$. For any fixed value of $Q^{2}$, conventional evolution of the structure function breaks down for $x<x_{0}\left(Q^{2}\right)$ where $x_{0}\left(Q^{2}\right)$ is calculable in the GDV/CDP. For values of $x<x_{0}\left(Q^{2}\right)$ "saturation" occurs in the sense of $\sigma_{\gamma^{*} p}\left(W^{2}, Q^{2}\right) / \sigma_{\gamma p}\left(W^{2}\right) \rightarrow 1$. Conversely, for any fixed $x \ll 0.1$, the conventional evolution holds for the gluon structure functions, provided $Q^{2}$ is sufficiently large.

A few concluding remarks will be given in section 7 .

\section{Two-gluon exchange in transverse-position- space representation and $J=1$}

The transverse-position-space representation [3, 2] for the total photoabsorption cross section,

$$
\sigma_{\gamma_{T, L}^{*} p}\left(W^{2}, Q^{2}\right)=\int d z \int d^{2} r_{\perp}\left|\psi_{T, L}\left(r_{\perp}, z(1-z), Q^{2}\right)\right|^{2} \sigma_{(q \bar{q}) p}\left(\vec{r}_{\perp}, W^{2}\right),
$$

and for diffractive forward production,

$$
\begin{aligned}
& \left.\frac{d \sigma_{\gamma_{T, L}^{*} p \rightarrow X p}\left(W^{2}, Q^{2}\right)}{d t}\right|_{t=0}=\frac{1}{16 \pi} \int d z \int d^{2} r_{\perp}\left|\psi_{T, L}\left(r_{\perp}, z(1-z), Q^{2}\right)\right|^{2} \\
& \sigma_{(q \bar{q}) p}^{2}\left(\vec{r}_{\perp}, W^{2}\right)
\end{aligned}
$$

conveniently summarizes the result of the $x \cong Q^{2} / W^{2} \rightarrow 0$ analysis of the twogluon-exchange dynamics. As intuitively suggested by the underlying $s$-channel point of view, we use the center-of-mass energy $W$ of the photon-proton (equivalently, of the $q \bar{q}$-proton) system as a variable in the dipole cross section. The assumed dependence on $W^{2}$ proved useful in the representation of the experimental data [5], as it automatically, and naturally, via $W^{2} \simeq Q^{2} / x$, induces scaling 
violations for the structure function $F_{2}\left(x, Q^{2}\right) \cong\left(Q^{2} / 4 \pi^{2} \alpha\right) \sigma_{\gamma^{*} p}\left(W^{2}, Q^{2}\right)$ as observed experimentally [16]. Frequently [13] $x$ replaces $W^{2}$ in (2.1), requiring a revision [14] of the original ansatz to incorporate scaling violations.

The wave function $\psi_{T, L}\left(\vec{r}_{\perp}, z(1-z), Q^{2}\right)$ describing the $\gamma^{*} q \bar{q}$ fluctuation of the virtual photon in (2.1) and (2.2) has the well-known form [2]

$$
\left|\psi_{T}\left(r_{\perp}, z(1-z), Q^{2}\right)\right|^{2}=\frac{6 \alpha}{(2 \pi)^{2}} \sum_{f} Q_{f}^{2}\left\{\left(z^{2}+(1-z)^{2}\right) \epsilon^{2} K_{1}\left(\epsilon r_{\perp}\right)^{2}+m_{f}^{2} K_{0}\left(\epsilon r_{\perp}\right)^{2}\right\}
$$

and

$$
\left|\psi_{L}\left(r_{\perp}, z(1-z), Q^{2}\right)\right|^{2}=\frac{6 \alpha}{(2 \pi)^{2}} \sum_{f} Q_{f}^{2} 4 Q^{2} z^{2}(1-z)^{2} K_{0}\left(\epsilon r_{\perp}\right)^{2}
$$

where

$$
\epsilon^{2}=z(1-z) Q^{2}+m_{f}^{2}
$$

In (2.3) to (2.5), $Q_{f}$ denotes the quark charge in units of $e, \alpha=e^{2} / 4 \pi$ the electromagnetic fine-structure constant, and $m_{f}$ denotes the quark mass. The functions $K_{0}\left(\epsilon r_{\perp}\right)$ and $K_{1}\left(\epsilon r_{\perp}\right)$ are modified Bessel functions.

It is important to emphasize that the justification for the use of the representations (2.1) and (2.2) as the $x \rightarrow 0$ limit of the two-gluon-exchange mechanism rests on applying them in conjunction with the representation for the color-dipole cross section given by [2, 5]

$$
\begin{aligned}
\sigma_{(q \bar{q}) p}\left(r_{\perp}, W^{2}\right) & =\int d^{2} l_{\perp} \tilde{\sigma}_{(q \bar{q}) p}\left(\vec{l}_{\perp}^{2}, W^{2}\right)\left(1-e^{-i \vec{l}_{\perp} \cdot \vec{r}_{\perp}}\right) \\
& =\sigma^{(\infty)} \cdot\left\{\begin{array}{cc}
\frac{1}{4} r_{\perp}^{2}\left\langle\vec{l}_{\perp}^{2}\right\rangle_{W^{2}}, & \text { for } \vec{r}^{2}\left\langle\vec{l}_{\perp}^{2}\right\rangle_{W^{2}} \rightarrow 0, \\
1 & \text { for } \vec{r}^{2} \rightarrow \infty,
\end{array}\right.
\end{aligned}
$$

where by definition

$$
\left\langle\vec{l}_{\perp}^{2}\right\rangle_{W^{2}}=\frac{\int d \vec{l}_{\perp}^{2} \vec{l}_{\perp}^{2} \tilde{\sigma}\left(\vec{l}_{\perp}^{2}, W^{2}\right)}{\int d \vec{l}_{\perp}^{2} \tilde{\sigma}\left(\vec{l}_{\perp}^{2}, W^{2}\right)}
$$

and

$$
\sigma^{(\infty)}=\pi \int d \vec{l}_{\perp}^{2} \tilde{\sigma}\left(\vec{l}_{\perp}^{2}, W^{2}\right)
$$

The limit of $\vec{r}_{\perp}^{2} \rightarrow 0$ corresponds to a vanishing interaction strength, due to color neutrality of the $q \bar{q}$ color-dipole state. The finite limit, $\sigma^{(\infty)}$, avoids an infinite 
color-dipole cross section in the limit of infinite quark-antiquark separation ${ }^{2}$, and it guarantees hadronic unitarity, provided $\sigma^{(\infty)}$ is well-behaved for $W \rightarrow \infty$.

For the ensuing examination of the spin properties of the $q \bar{q}$ states, it proves useful to introduce the variable

$$
\vec{r}_{\perp}^{\prime}=\sqrt{z(1-z)} \vec{r}
$$

and to first of all consider the integral over $d^{2} r_{\perp}^{\prime}$ of the $q \bar{q}$-vacuum-polarization loop by itself. ${ }^{3}$ We restrict ourselves to a vanishing quark mass, $m_{f}=0 .{ }^{4}$ In terms of $\vec{r}_{\perp}^{\prime}$ from (2.9), we have

$$
\begin{aligned}
\int d^{2} r_{\perp}\left|\psi_{T}\left(r_{\perp}, z(1-z), Q^{2}\right)\right|^{2} & =\int \frac{d^{2} r_{\perp}^{\prime}}{z(1-z)}\left|\psi_{T}\left(\frac{r_{\perp}^{\prime}}{\sqrt{z(1-z)}}, z(1-z), Q^{2}\right)\right|^{2} \\
& =\frac{6 \alpha}{(2 \pi)^{2}} Q^{2} \sum_{f} Q_{f}^{2}\left(z^{2}+(1-z)^{2}\right) \int d^{2} r_{\perp}^{\prime} K_{1}\left(\sqrt{Q^{2}} r_{\perp}^{\prime}\right)^{2}
\end{aligned}
$$

and

$$
\begin{aligned}
\int d^{2} r_{\perp}\left|\psi_{L}\left(r_{\perp}, z(1-z), Q^{2}\right)\right|^{2} & =\int \frac{d^{2} r_{\perp}^{\prime}}{z(1-z)}\left|\psi_{L}\left(\frac{r_{\perp}^{\prime}}{\sqrt{z(1-z)}}, z(1-z), Q^{2}\right)\right|^{2} \\
& =\frac{6 \alpha}{(2 \pi)^{2}} Q^{2} \sum_{f} Q_{f}^{2} z(1-z) \int d^{2} r_{\perp}^{\prime} K_{0}\left(\sqrt{Q^{2}} r_{\perp}^{\prime}\right)^{2} .
\end{aligned}
$$

The $z$-dependence in (2.10) and (2.11) that originates from the $\gamma^{*} q \bar{q}$ coupling is immediately seen to be characteristic of the spin $J=1$ nature of the photon. It coincides with the angular dependence in the $q \bar{q}$ rest frame well-known from e.g. $e^{+} e^{-}$annihilation into $q \bar{q}$. Indeed, upon identifying

$$
\begin{aligned}
\sin ^{2} \theta & =4 z(1-z), \\
\cos \theta & =1-2 z,
\end{aligned}
$$

\footnotetext{
${ }^{2}$ It is worth noting that the energy dependence of the color-dipole cross section in the GVD/CDP [5] enters exclusively via a (soft) increase of the gluon-transverse-momentum transfer, $\vec{l}_{\perp}$, with energy, i.e., via $\tilde{\sigma}_{(q \bar{q}) p}\left(\vec{l}_{\perp}^{2}\right)=\tilde{\sigma}_{(q \bar{q}) p}\left(\vec{l}^{2}\left(W^{2}\right)\right)$.

${ }^{3}$ The relevance of hadronic vacuum polarization for photo- and electroproduction (from nuclei) at low $x$ during the pre-QCD era was stressed in particular by V. Gribov [17.

${ }^{4}$ In applying the approach based on (2.1) to the description of the experimental data, it proved useful [5] to return to momentum space and to introduce an effective constituent-quark mass that coincides with the value suggested by quark-hadron duality 18 in $e^{+} e^{-}$-annihilation. This mass then effectively provides a lower limit in the integration over the mass spectrum of the $q \bar{q}$ vector states the photon virtually dissociates into.
} 
we may represent the integrations over $d z$ in (2.1) in terms of $\cos \theta$ and the rotation function $d_{\lambda, \mu}^{1}(z)$,

$$
\begin{aligned}
d_{1,1}^{1}(z) & =\frac{1}{2}(1+\cos \theta)=1-z \\
d_{1,-1}^{1}(z) & =\frac{1}{2}(1-\cos \theta)=z \\
d_{1,0}^{1}(z) & =\frac{1}{\sqrt{2}} \sin \theta=\sqrt{2} \sqrt{z(1-z)} .
\end{aligned}
$$

The integrations over $d z$ in (2.1) and (2.2), as far as the photon wave functions are concerned, according to (2.10) and (2.11) can then be written as

$$
\begin{aligned}
d z \cdot\left(z^{2}+(1-z)^{2}\right) & ==-\frac{1}{2} d \cos \theta \cdot \frac{1}{2}\left(1+\cos ^{2} \theta\right) \\
& =d z \cdot\left[\left|d_{1,1}^{1}(z)\right|^{2}+\left|d_{1,-1}^{1}(z)\right|^{2}\right]
\end{aligned}
$$

and

$$
\begin{aligned}
d z \cdot z(1-z) & =-\frac{1}{2} d \cos \theta \cdot \frac{1}{4} \sin ^{2} \theta \\
& =d z \cdot \frac{1}{2}\left|d_{1,0}^{1}(z)\right|^{2}
\end{aligned}
$$

In (2.14), we recognize the $\left(1+\cos ^{2} \theta\right)$ distribution from $e^{+} e^{-}$annihilation into $q \bar{q}$.

Returning to the virtual photoabsorption cross section (2.1) and introducing $\vec{r}_{\perp}^{\prime}$, we have

$$
\begin{aligned}
& \sigma_{\gamma_{T, L}^{*} p}\left(W^{2}, Q^{2}\right) \\
& =\int d z \int \frac{d^{2} r_{\perp}^{\prime}}{z(1-z)}\left|\psi_{T, L}\left(\frac{r_{\perp}^{\prime}}{\sqrt{z(1-z)}}, z(1-z), Q^{2}\right)\right|^{2} \sigma_{(q \bar{q}) p}\left(\frac{r_{\perp}^{\prime}}{\sqrt{z(1-z)}}, W^{2}\right),
\end{aligned}
$$

where according to (2.6)

$$
\begin{aligned}
& \sigma_{(q \bar{q}) p}\left(\frac{r_{\perp}^{\prime}}{\sqrt{z(1-z)}}, W^{2}\right) \\
& =\int d^{2} l_{\perp}^{\prime} z(1-z) \tilde{\sigma}_{(q \bar{q}) p}\left(\vec{l}_{\perp}^{\prime 2} z(1-z), W^{2}\right)\left(1-e^{-i \vec{l}_{\perp}^{\prime} \vec{r}_{\perp}^{\prime}}\right) .
\end{aligned}
$$

In (2.17), we use

$$
\vec{l}_{\perp}^{\prime}=\frac{\vec{l}_{\perp}}{\sqrt{z(1-z)}}
$$


Reading (2.16) in conjunction with (2.10) and (2.11) as well as (2.17), we note the appearance of products of $d_{\lambda, \mu}^{1}(z)$ functions in (2.16). In order to take advantage of their orthogonality properties, we expand the $(q \bar{q}) p \rightarrow(q \bar{q}) p$ amplitude in (2.17) ${ }^{5}$ in terms of states of different spin $J$ of the $q \bar{q}$ system, i.e. in terms of $\bar{\sigma}_{(q \bar{q})_{T, L}^{J} p}\left(\vec{l}_{\perp}^{\prime 2}, W^{2}\right)$. Accordingly,

$$
\begin{aligned}
& (1-z)\left[z(1-z) \tilde{\sigma}_{(q \bar{q}) p}\left(\vec{l}_{\perp}^{\prime 2} z(1-z), W^{2}\right)\right] \\
= & d_{11}^{1}(z) \bar{\sigma}_{(q \bar{q})_{T}^{J=1} p}\left(\vec{l}_{\perp}^{\prime 2}, W^{2}\right)+d_{11}^{2}(z) \bar{\sigma}_{(q \bar{q})_{T}^{J=2} p}\left(\vec{l}_{\perp}^{\prime 2}, W^{2}\right)+\cdots, \\
& z\left[z(1-z) \tilde{\sigma}_{(q \bar{q}) p}\left(\vec{l}_{\perp}^{\prime 2} z(1-z), W^{2}\right)\right] \\
= & d_{1-1}^{1}(z) \bar{\sigma}_{(q \bar{q})_{T}^{J=1} p}\left(\vec{l}_{\perp}^{\prime 2}, W^{2}\right)-d_{1-1}^{2}(z) \bar{\sigma}_{(q \bar{q})_{T}^{J=2} p}\left(\vec{l}_{\perp}^{\prime 2}, W^{2}\right)+\cdots
\end{aligned}
$$

and

$$
\begin{aligned}
& \sqrt{2} \sqrt{z(1-z)} z(1-z) \tilde{\sigma}_{(q \bar{q}) p}\left(\vec{l}_{\perp}^{\prime 2} z(1-z), W^{2}\right) \\
= & d_{10}^{1}(z) \bar{\sigma}_{(q \bar{q})_{L}^{J=1} p}\left(\vec{l}_{\perp}^{\prime 2}, W^{2}\right)+d_{10}^{2}(z) \bar{\sigma}_{(q \bar{q})_{L}^{J=2} p}\left(\vec{l}_{\perp}^{\prime 2}, W^{2}\right)+\cdots .
\end{aligned}
$$

Inserting (2.19) and (2.20), respectively, into (2.17) and subsequently (2.17) into (2.16), we note that indeed only contributions due to elastic interactions $(q \bar{q})_{T, L}^{J=1} p \rightarrow(q \bar{q})_{T, L}^{J=1} p$, with the proton remain, and (2.16) becomes

$\sigma_{\gamma_{T, L}^{*} p}\left(W^{2}, Q^{2}\right)=\int d z \int \frac{d^{2} r_{\perp}^{\prime}}{z(1-z)}\left|\psi_{T, L}\left(\frac{r_{\perp}^{\prime}}{\sqrt{z(1-z)}}, z(1-z), Q^{2}\right)\right|^{2} \sigma_{(q \bar{q})_{T, L}^{J=1} p}\left(\vec{r}_{\perp}^{\prime}, W^{2}\right)$.

According to (2.17), the cross sections $\sigma_{(q \bar{q})_{T, L}^{J=1} p}\left(\vec{r}_{\perp}^{\prime}, W^{2}\right)$ in (2.21), corresponding to the (imaginary parts of the) elastic forward-scattering amplitudes $(q \bar{q})_{T, L}^{J=1} p \rightarrow$ $(q \bar{q})_{T, L}^{J=1} p$, are given by

$$
\sigma_{(q \bar{q})_{T, L}^{J=1} p}\left(\vec{r}_{\perp}^{\prime}, W^{2}\right)=\int d^{2} l_{\perp}^{\prime} \bar{\sigma}_{(q \bar{q})_{T, L}^{J=1} p}\left(\vec{l}_{\perp}^{\prime 2}, W^{2}\right)\left(1-e^{-i \vec{l}_{\perp}^{\prime} \cdot \vec{r}_{\perp}^{\prime}}\right),
$$

where $\bar{\sigma}_{q \bar{q}) J=1}^{J=1} p\left(\vec{l}_{\perp}^{\prime 2}, W^{2}\right)$ according to (2.19) and (2.20) is explicitly given by

$$
\begin{aligned}
\bar{\sigma}_{(q \bar{q})_{T}^{J=1} p}\left(\vec{l}_{\perp}^{\prime 2}, W^{2}\right) & =3 \int d z z(1-z)^{3} \tilde{\sigma}_{(q \bar{q}) p}\left(\vec{l}_{\perp}^{\prime 2} z(1-z), W^{2}\right) \\
& =3 \int d z z^{3}(1-z) \tilde{\sigma}_{(q \bar{q}) p}\left(\vec{l}_{\perp}^{\prime 2} z(1-z), W^{2}\right)
\end{aligned}
$$

${ }^{5}$ Actually, we expand the amplitude for $(q \bar{q})^{J=1} p \rightarrow(q \bar{q})^{J} p$ in terms of the spin $J$ of the outgoing $(q \bar{q})$ state. This is seen in (2.19) and (2.20), where $z(1-z) \tilde{\sigma}\left(\vec{l}_{\perp}^{\prime 2} z(1-z), W^{2}\right)$ is multiplied by an appropriate projection factor stemming from the substitution of (2.17) into (2.16). 
and

$$
\bar{\sigma}_{(q \bar{q})_{L}^{J=1} p}\left(\vec{l}_{\perp}^{\prime 2}, W^{2}\right)=6 \int d z(z(1-z))^{2} \tilde{\sigma}_{(q \bar{q}) p}\left(\vec{l}_{\perp}^{\prime 2} z(1-z), W^{2}\right)
$$

We stress that (2.21) with (2.22) is no less general than (2.1) with (2.6). The difference between these two representations is only due to the exploitation of the decomposition of the $(q \bar{q})^{J=1} p \rightarrow(q \bar{q})^{J} p$ amplitude into contributions from different spin $J$ of the $q \bar{q}$ system.

The representation (2.1) with (2.6) for the total cross section $\sigma_{\gamma_{T, L}^{*}}\left(W^{2}, Q^{2}\right)$ contains redundant and irrelevant contributions on the right-hand side due to $(q \bar{q})^{J=1} p \rightarrow(q \bar{q})^{J \neq 1} p$ scattering amplitudes. These contributions vanish upon integration, and they are eliminated right from the beginning in the representation (2.21) with (2.22).

Fits to the experimental data for $\sigma_{\gamma_{T, L}^{*}}{ }\left(W^{2}, Q^{2}\right)$ are frequently based [12, 13, 14] on (2.1). The representation

$$
\sigma_{\gamma_{T, L}^{*} p}\left(W^{2}, Q^{2}\right)=\int d z \int d^{2} r_{\perp}\left|\psi_{T, L}\left(r_{\perp}, z(1-z), Q^{2}\right)\right|^{2} \sigma_{(q \bar{q}) p}\left(\vec{r}_{\perp}, W^{2}\right)
$$

is used for the fit [12, 13, 14] upon adopting an ansatz for the color-dipole cross section, $\sigma_{(q \bar{q}) p}$, as a function of $r_{\perp}$ and either $W^{2}$ [12], or alternatively, $x$ [13]. Different functional forms for $\sigma_{(q \bar{q}) p}$ will in general yield equally good fits for their specific sets of fit parameters, provided the different functional forms agree in their $(q \bar{q})^{J=1} p \rightarrow(q \bar{q})^{J=1} p$ content. It is of no surprise that widely differing fit results [15] for color-dipole cross sections were extracted by different authors; dipolecross sections that implicitly contain approximately identical $(q \bar{q})^{J=1}$ but widely differing $(q \bar{q})^{J \neq 1}$ final-state contributions obviously yield identical representations of the total cross section, $\sigma_{\gamma^{*} p}\left(W^{2}, Q^{2}\right)$.

Such ambiguities are avoided in the representation (2.21) with (2.22). For the sake of clarity, it may be appropriate to equivalently express (2.21) in terms of the original variable $r_{\perp}$,

$$
\begin{aligned}
& \sigma_{\gamma_{T, L}^{*} p}\left(W^{2}, Q^{2}\right)= \\
& =\int d z \int d^{2} r_{\perp}\left|\psi_{T, L}\left(r_{\perp}, z(1-z), Q^{2}\right)\right|^{2} \sigma_{(q \bar{q})_{T, L}^{J=1} p}\left(\vec{r}_{\perp} \sqrt{z(1-z)}, W^{2}\right)
\end{aligned}
$$


where according to (2.22),

$$
\sigma_{(q \bar{q})_{T, L}^{J=1}}\left(\vec{r}_{\perp} \sqrt{z(1-z)}, W^{2}\right)=\int d^{2} l_{\perp}^{\prime} \bar{\sigma}_{(q \bar{q})_{T, L}^{J=1} p}\left(\vec{l}_{\perp}^{\prime 2}, W^{2}\right)\left(1-e^{-i \vec{l}_{\perp}^{\prime} \cdot \vec{r}_{\perp} \sqrt{z(1-z)}}\right) .
$$

In distinction from (2.25), in (2.26) the variable $r_{\perp}^{\prime}=r_{\perp} \cdot \sqrt{z(1-z)}$ appears as argument of the dipole cross section, and this is sufficient to assure that only $(q \bar{q})^{J=1} p \rightarrow(q \bar{q})^{J=1} p$ is included.

A few additional comments on the form (2.21) for $\sigma_{\gamma_{T, L}^{*}}$ may be appropriate. The dipole cross section in (2.21) depends only on $\vec{r}^{\prime}$ and $W^{2}$, while the dependence on $Q^{2}$ and the $q \bar{q}$ configuration variable $z$ is transferred to the photon wave function thus describing the $q \bar{q}$ fluctuation of the photon with the appropriate $z$ dependence as in $e^{+} e^{-}$annihilation; compare the second equality in (2.10) and (2.11). Once $\sigma_{(q \bar{q})^{J=1}}\left(r_{\perp}^{\prime}, W^{2}\right)$ is determined from a fit to the experimental data for $\sigma_{\gamma_{T, L}^{*}}\left(W^{2}, Q^{2}\right)$, it may be inserted into the cross section for diffractive production (2.2) via

$$
\sigma_{(q \bar{q}) p}\left(\vec{r}_{\perp}, W^{2}\right) \rightarrow \sigma_{(q \bar{q})_{T, L}^{J=1} p}\left(\vec{r}_{\perp} \sqrt{z(1-z)}, W^{2}\right)
$$

to obtain a unique prediction for "elastic" diffractive forward production

$$
\gamma_{T, L}^{*} p \rightarrow(q \bar{q})_{T, L}^{J=1} p
$$

of $q \bar{q}$ vector states. It is diffractive production of vector states that is uniquely connected with the total cross section $\sigma_{\gamma^{*} p}\left(W^{2}, Q^{2}\right)$ at low $x$. Besides the $J=1$ diffractive continuum, via quark-hadron duality [18, 19], it is vector meson production in particular that is uniquely related to the total cross section $\sigma_{\gamma_{T, L}^{*}}\left(W^{2}, Q^{2}\right)$.

Diffractive production in general contains a large part of "inelastic" diffraction, ${ }^{6}$

$$
\gamma_{T, L}^{*} p \rightarrow(q \bar{q})^{J \neq 1} p,
$$

that remains unconstrained by fits of the dipole cross section to $\sigma_{\gamma_{T, L}^{*} p}\left(W^{2}, Q^{2}\right)$. A description of the sum of elastic and inelastic diffractive production according

\footnotetext{
${ }^{6}$ Compare 20 for a treatment of the total cross section and of diffraction based on an elastic and inelastic component.
} 
to (2.2) must contain an additive component in the dipole cross section that is projected to zero and thus remains inert when included in (2.1).

In short, if (2.21) with (2.22) is used to describe the total cross section, a successful description will imply a prediction for elastic diffraction, (2.29). If (2.1) with (2.6) is employed to describe the total cross section by fitting an ansatz for the dipole cross section, it is by no means guaranteed that this fit, when substituted into (2.2), will be relevant for a representation of elastic as well as inelastic diffractive production. After all, as repeatedly stressed, inelastic diffraction remains unconstrained by $\sigma_{\gamma_{T, L}^{*}}\left(W^{2}, Q^{2}\right)$. The use of the form (2.1) for the total cross section necessitates a simultaneous fit to both, the total cross section (2.1) and (the sum of elastic and inelastic) diffractive production according to (2.2). In principle $^{7}$, such a fit will provide a unique color-dipole cross section that in addition to $\sigma_{\gamma_{T, L}^{*}} p\left(W^{2}, Q^{2}\right)$ describes both elastic and inelastic diffraction. If the fit to diffraction is achieved by an additive contribution [12, 13, 14] in the dipole cross section relative to the one successfully used in the fit to $\sigma_{\gamma_{T, L}^{*}}\left(W^{2}, Q^{2}\right)$, it ought to be verified that the added term only contributes to inelastic diffraction while leaving the (previous) fit to $\sigma_{\gamma_{T, L}^{*}}\left(W^{2}, Q^{2}\right)$ unchanged.

\section{Momentum space representation and sum rule.}

The connection between the total cross section and elastic diffraction becomes explicit in terms of a sum rule [4] that relates the total cross section to elastic diffractive forward production.

In momentum space, the $x \rightarrow 0$ representation of the total cross section (2.1) becomes 4

$$
\begin{aligned}
\sigma_{\gamma_{T, L}^{*} p}\left(W^{2}, Q^{2}\right)= & \frac{3}{16 \pi^{3} \cdot 2} \int d z \int d^{2} k_{\perp} \int d^{2} l_{\perp} \tilde{\sigma}_{(q \bar{q}) p}\left(\vec{l}_{\perp}^{2}, W^{2}\right) \\
& \cdot\left|\mathcal{M}_{T, L}\left(z, \vec{k}_{\perp}, Q^{2}\right)-\mathcal{M}_{T, L}\left(z, \vec{k}_{\perp}+\vec{l}_{\perp}, Q^{2}\right)\right|^{2}
\end{aligned}
$$

The representation (3.1) is related to the representation in transverse position

\footnotetext{
${ }^{7}$ Since the data on diffraction do not reach the accuracy of the data for $\sigma_{\gamma^{*} p}$, it seems preferable to fit $\sigma_{\gamma^{*} p}$ according to (2.21) with (2.22) and compare with elastic diffraction (into continuum states as well as vector-meson production) and subsequently fit inelastic diffraction by introducing an (orthogonal) additive contribution.
} 
space (2.1) by the substitution of the photon wave function in momentum space that is given by

$$
\begin{aligned}
& \sum_{\lambda, \lambda^{\prime}}\left|\psi_{T, L}^{\left(\lambda, \lambda^{\prime}\right)}\left(\vec{r}_{\perp}, z ; Q^{2}\right)\right|^{2}=3 \cdot \frac{4 \pi}{\left(16 \pi^{3}\right)^{2}} \int d^{2} k_{\perp}^{\prime} \int d^{2} k_{\perp} \mathcal{M}_{T, L}^{*}\left(\vec{k}_{\perp}^{\prime}, z, Q^{2}\right) \\
& \mathcal{M}_{T, L}\left(\vec{k}_{\perp}, z, Q^{2}\right) \exp \left(i \vec{k}_{\perp}^{\prime}-\vec{k}_{\perp}\right) \vec{r}_{\perp}
\end{aligned}
$$

with

$$
\mathcal{M}_{T}^{*}\left(\vec{k}_{\perp}^{\prime}, z, Q^{2}\right) \cdot \mathcal{M}_{T}\left(\vec{k}_{\perp}, z, Q^{2}\right)=\frac{8 \pi \alpha\left(\vec{k}_{\perp}^{\prime} \cdot \vec{k}_{\perp}\right) \sum_{f} Q_{f}^{2}\left(z^{2}+(1-z)^{2}\right)}{\left(z(1-z) Q^{2}+\vec{k}_{\perp}^{\prime 2}\right)\left(z(1-z) Q^{2}+\vec{k}_{\perp}^{2}\right)}
$$

and

$$
\mathcal{M}_{L}^{*}\left(\vec{k}_{\perp}^{\prime}, z, Q^{2}\right) \cdot \mathcal{M}_{L}\left(\vec{k}_{\perp}, z, Q^{2}\right)=\frac{32 \pi \alpha Q^{2} \sum_{f} Q_{f}^{2} z^{2}(1-z)^{2}}{\left(z(1-z) Q^{2}+\vec{k}_{\perp}^{\prime 2}\right)\left(z(1-z) Q^{2}+\vec{k}_{\perp}^{2}\right)} .
$$

In (3.1),$\vec{k}_{\perp}$ denotes the transverse momentum of the quark. It is related to the mass, $M$, of the $q \bar{q}$ state by

$$
M^{2}=\frac{\vec{k}_{\perp}^{2}+m^{2}}{z(1-z)}
$$

where the quark mass $m$ will be put to $m=0$ for simplicity. After one angular integration, and upon introducing $M^{2}$ as integration variable, via

$$
d^{2} k_{\perp}=\frac{1}{2} z(1-z) d M^{2} d \varphi
$$

(3.1) becomes $^{8}$

$$
\begin{aligned}
& \sigma_{\gamma_{T}^{*} p}\left(W^{2}, Q^{2}\right)=\frac{c_{0}^{2}}{64 \pi} \int_{m_{0}^{2}} d M^{2} \frac{M^{2}}{\left(Q^{2}+M^{2}\right)^{2}} \int d z\left(z^{2}+(1-z)^{2}\right) \\
& \cdot \int d^{2} l_{\perp}^{\prime} z(1-z) \tilde{\sigma}_{(q \bar{q}) p}\left(\vec{l}_{\perp}^{\prime 2} z(1-z), W^{2}\right) F_{T}\left(Q^{2}, M^{2}, \vec{l}_{\perp}^{\prime 2}\right),
\end{aligned}
$$

and

$$
\begin{aligned}
& \sigma_{\gamma_{L}^{*} p}\left(W^{2}, Q^{2}\right)=\frac{c_{0}^{2}}{16 \pi} \int_{m_{0}^{2}} d M^{2} \frac{Q^{2}}{\left(Q^{2}+M^{2}\right)^{2}} \int d z z(1-z) \\
& \cdot \int d^{2} l_{\perp}^{\prime} z(1-z) \tilde{\sigma}_{(q \bar{q}) p}\left(\vec{l}_{\perp}^{\prime 2} z(1-z), W^{2}\right) \cdot F_{L}\left(Q^{2}, M^{2}, \vec{l}_{\perp}^{\prime 2}\right) .
\end{aligned}
$$

\footnotetext{
${ }^{8}$ Note that we introduce [5] the threshold mass $m_{0}$, as announced in the footnote in connection with (2.10). We ignore the additive "correction terms" given in [5, 4] that assure an identical threshold mass, $m_{0}$ for the incoming and outgoing $q \bar{q}$ pair in the forward Compton amplitude.
} 
Here,

$$
c_{0}=2 \sqrt{\frac{2 N_{c}}{\pi}} \sqrt{\frac{4 \pi \alpha R_{e^{+} e^{-}}}{3}} .
$$

The number of quark colors is $N_{c}=3$, and $R_{e^{+} e^{-}}$denotes the cross section for $e^{+} e^{-}$annihilation, $e^{+} e^{-} \rightarrow q \bar{q} \rightarrow$ hadrons, in units of the cross section for $e^{+} e^{-} \rightarrow \mu^{+} \mu^{-}$,

$$
R_{e^{+} e^{-}}=3 \sum_{f} Q_{f}^{2}
$$

The sum runs over the quark charges, $Q_{f}$, in units of $e$. The functions $F_{T}\left(Q^{2}, M^{2}, \vec{l}_{\perp}^{\prime 2}\right)$ and $F_{L}\left(Q^{2}, M^{2}, \vec{l}_{\perp}^{\prime 2}\right)$ in (3.7) and (3.8) are given by

$$
F_{T}\left(Q^{2}, M^{2}, \vec{l}_{\perp}^{\prime 2}\right)=1-\frac{Q^{2}+M^{2}}{2 M^{2}}\left(1+\frac{M^{2}-Q^{2}-\vec{l}_{\perp}^{\prime 2}}{\sqrt{\left(Q^{2}+M^{2}+\vec{l}_{\perp}^{\prime 2}\right)^{2}-4 M^{2} \vec{l}_{\perp}^{\prime 2}}}\right)
$$

and

$$
F_{L}\left(Q^{2}, M^{2}, \vec{l}_{\perp}^{\prime 2}\right)=1-\frac{Q^{2}+M^{2}}{\sqrt{\left(M^{2}+Q^{2}+\vec{l}_{\perp}^{\prime 2}\right)^{2}-4 M^{2} \vec{l}_{\perp}^{\prime 2}}} .
$$

The $z$-dependence in (3.7) and (3.8) is identical to the one encountered in transverseposition-space. As in the transverse-position-space treatment, we now use (2.19) and (2.20) and express the transverse and the longitudinal photoabsorption cross section in (3.7) and (3.8), respectively, in terms of $\bar{\sigma}_{(q \bar{q})_{T, L}^{J=1} p}\left(\vec{l}^{\prime 2}, W^{2}\right)$ from (2.23) and (2.24). We obtain

$$
\begin{aligned}
& \sigma_{\gamma_{T}^{*} p}\left(W^{2}, Q^{2}\right)=\frac{c_{0}^{2}}{64 \pi} \int_{m_{0}^{2}} d M^{2} \frac{M^{2}}{\left(Q^{2}+M^{2}\right)^{2}} \cdot \\
& \cdot \int d z\left(z^{2}+(1-z)^{2}\right) \int d^{2} l_{\perp}^{\prime} \bar{\sigma}_{(q \bar{q})_{T}^{J=1} p}\left(\vec{l}_{\perp}^{\prime 2}, W^{2}\right) F_{T}\left(Q^{2}, M^{2}, \vec{l}_{\perp}^{\prime 2}\right)
\end{aligned}
$$

and

$$
\begin{aligned}
& \sigma_{\gamma_{L}^{*} p}\left(W^{2}, Q^{2}\right)=\frac{c_{0}^{2}}{16 \pi} \int_{m_{0}^{2}} d M^{2} \frac{Q^{2}}{\left(Q^{2}+M^{2}\right)^{2}} \cdot \\
& \cdot \int d z z(1-z) \int d^{2} l_{\perp}^{\prime} \bar{\sigma}_{(q \bar{q})_{L}^{J=1} p}\left(\vec{l}_{\perp}^{\prime 2}, W^{2}\right) F_{L}\left(Q^{2}, M^{2}, \vec{l}_{\perp}^{\prime 2}\right) .
\end{aligned}
$$

The integrations over $z$ in (3.13) and (3.14) can be carried out immediately to yield the factors $2 / 3$ and $1 / 6$, respectively. 
The results (3.13) and (3.14) may be conveniently summarized by the substitution rule

$$
z(1-z) \tilde{\sigma}_{(q \bar{q}) p}\left(\vec{l}_{\perp}^{\prime 2} z(1-z), W^{2}\right) \rightarrow \bar{\sigma}_{(q \bar{q})_{T, L}^{J=1} p}\left(\vec{l}_{\perp}^{\prime 2}, W^{2}\right)
$$

to be applied in (3.7) and (3.8). We note that (3.13) and (3.14) are the analogue of the transverse-position-space result (2.21). According to (3.13) and (3.14), the total cross section, $\sigma_{\gamma_{T, L}^{*} p}\left(W^{2}, Q^{2}\right)$, or equivalently the virtual forward Compton scattering amplitude, for $x \ll 1$, is determined by $(q \bar{q})_{T, L}^{J=1} p \rightarrow(q \bar{q})_{T, L}^{J=1} p$ forward scattering.

We turn to diffractive production and the derivation of the sum rule. We substitute the momentum-space expressions of the photon-wave functions (3.2) to (3.4) into (2.2), as well as the dipole cross section (2.6). Introducing $M^{2}$ from (3.5) the cross section for diffractive production, $\gamma_{T, L}^{*} p \rightarrow X_{T, L} p$, reads

$$
\begin{aligned}
& \left.\frac{d \sigma_{\gamma_{T, L}^{*} p \rightarrow X_{T, L} p}}{d t d z d M^{2}}\right|_{t=0}=\frac{3}{2} \frac{1}{\left(16 \pi^{2}\right)^{2}} \\
& \cdot z(1-z) \int d \varphi\left[\int d^{2} l_{\perp} \tilde{\sigma}_{(q \bar{q}) p}\left(\vec{l}_{\perp}^{2}, W^{2}\right)\left(\mathcal{M}_{T, L}\left(\vec{k}_{\perp}, z, Q^{2}\right)-\mathcal{M}_{T, L}\left(\vec{k}_{\perp}+\vec{l}_{\perp}, z, Q^{2}\right)\right)\right]^{2} .
\end{aligned}
$$

Upon angular integration, (3.16) for transverse and longitudinal photons respectively, leads to

$$
\begin{aligned}
& \sqrt{\left.\frac{d \sigma_{\gamma_{T}^{*} p \rightarrow X p}}{d z d t d M^{2}}\right|_{t=0}}=\frac{c_{0}}{32 \pi} \sqrt{z^{2}+(1-z)^{2}} \frac{M}{Q^{2}+M^{2}} \cdot \\
& \cdot \int d^{2} l_{\perp}^{\prime} z(1-z) \tilde{\sigma}\left(\vec{l}_{\perp}^{\prime 2} z(1-z), W^{2}\right) F_{T}\left(Q^{2}, M^{2}, \vec{l}_{\perp}^{\prime 2}\right),
\end{aligned}
$$

and

$$
\begin{aligned}
& \sqrt{\left.\frac{d \sigma_{\gamma_{L}^{*} p \rightarrow X p}}{d z d t d M^{2}}\right|_{t=0}}=\frac{c_{0}}{16 \pi} \sqrt{z(1-z)} \frac{\sqrt{Q^{2}}}{Q^{2}+M^{2}} . \\
& \cdot \int d^{2} l_{\perp}^{\prime} z(1-z) \tilde{\sigma}\left(\vec{l}_{\perp}^{\prime 2} z(1-z), W^{2}\right) F_{L}\left(Q^{2}, M^{2}, \vec{l}_{\perp}^{\prime 2}\right) .
\end{aligned}
$$

Finally, we compare (3.17) and (3.18) with (3.7) and (3.8) to obtain the sum rules

$$
\begin{aligned}
& \sigma_{\gamma_{T}^{*} p}\left(W^{2}, Q^{2}\right)=\frac{c_{0}}{2} \int_{m_{0}^{2}} d M^{2} \frac{M}{Q^{2}+M^{2}} \\
& \cdot \int d z \sqrt{z^{2}+(1-z)^{2}} \sqrt{\left.\frac{d \sigma_{\gamma_{T}^{*} p \rightarrow X_{T, L} p}}{d z d t d M^{2}}\right|_{t=0}}
\end{aligned}
$$


and

$$
\begin{gathered}
\sigma_{\gamma_{L}^{*} p}\left(W^{2}, Q^{2}\right)=c_{0} \int_{m_{0}^{2}} d M^{2} \frac{\sqrt{Q^{2}}}{Q^{2}+M^{2}} \\
\cdot \int d z \sqrt{z(1-z)} \sqrt{\left.\frac{d \sigma_{\gamma_{L}^{*} p \rightarrow X_{T, L} p}}{d z d t d M^{2}}\right|_{t=0}} .
\end{gathered}
$$

Note that the diffractive production cross sections in general involve final states $X$ that contain $(q \bar{q})^{J}$ states of arbitrary spin $J$. The corresponding distribution in $z$ should appear as angular distribution of a quark and an antiquark jet in the rest frame of the $(q \bar{q})^{J}$ system. The $z$-dependent projections in (3.19) and (3.20), according to (2.14) and (2.15), project on $J=1$ final states, $(q \bar{q})^{J=1}$.

We may rewrite (3.19) and (3.20) as ${ }^{9}$

$$
\sigma_{\gamma_{T}^{*} p}\left(W^{2}, Q^{2}\right)=\sqrt{16 \pi} \sqrt{\frac{\alpha R_{e^{+} e^{-}}}{3 \pi}} \int_{m_{0}^{2}} d M^{2} \frac{M}{Q^{2}+M^{2}} \sqrt{\left.\frac{d \sigma_{\gamma_{T}^{*} p \rightarrow X_{T}^{J=1} p}}{d t d M^{2}}\right|_{t=0}}
$$

and

$$
\sigma_{\gamma_{L}^{*} p}\left(W^{2}, Q^{2}\right)=\sqrt{16 \pi} \sqrt{\frac{\alpha R_{e^{+} e^{-}}}{3 \pi}} \int_{m_{0}^{2}} d M^{2} \frac{\sqrt{Q^{2}}}{Q^{2}+M^{2}} \sqrt{\left.\frac{d \sigma_{\gamma_{L}^{*} p \rightarrow X_{L}^{J}=1} p}{d t d M^{2}}\right|_{t=0}} .
$$

The sum rules (3.21) and (3.22) express the total cross section in terms of elastic diffraction, $\gamma_{T, L}^{*} p \rightarrow X_{T, L}^{J=1} p$, where $X_{T, L}^{J=1}$ stands for $X_{T, L}^{J=1} \equiv(q \bar{q})_{T, L}^{J=1}$. Explicitly, the diffractive-production cross sections in (3.21) and (3.22) are given by

$$
\left.\frac{d \sigma_{\gamma_{T}^{*} p \rightarrow X_{T}^{J=1} p}}{d t d M^{2}}\right|_{t=0}=\left(\frac{c_{0}}{32 \pi}\right)^{2} \frac{M^{2}}{\left(M^{2}+Q^{2}\right)^{2}} \frac{2}{3}\left|\int d^{2} l^{\prime} \bar{\sigma}_{(q \bar{q})_{T}^{J=1}}\left(\vec{l}_{\perp}^{2}, W^{2}\right) F_{T}\left(Q^{2}, M^{2}, \vec{l}_{\perp}^{2}\right)\right|^{2},
$$

and

$$
\left.\frac{d \sigma_{\gamma_{L}^{*} p \rightarrow X_{L}^{J=1} p}}{d t d M^{2}}\right|_{t=0}=\left(\frac{c_{0}}{16 \pi}\right)^{2} \frac{Q^{2}}{\left(M^{2}+Q^{2}\right)^{2}} \frac{1}{6}\left|\int d^{2} l^{\prime} \bar{\sigma}_{(q \bar{q})_{L}^{J=1}}\left(\vec{l}_{\perp}^{2}, W^{2}\right) F_{L}\left(Q^{2}, M^{2}, \vec{l}_{\perp}^{2}\right)\right|^{2} .
$$

They are related to (3.17) and (3.18) by the substitution rule (3.15) with subsequent integration over $z$.

\footnotetext{
${ }^{9}$ The sum rules (3.21) and (3.22) are identical to the ones given in 4]. As repeatedly stressed, in [4] they were obtained upon introducing a specific ansatz for the color-dipole cross section [5], while the present derivation is entirely based on the two-gluon-exchange generic structure.
} 
In summary, solely based on the structure of the two-gluon-exchange dynamics of fig. 1, we derived the sum rules (3.21) and (3.22). They relate the transverse and longitudinal part of the total photoabsorption cross section to diffractive forward production of $q \bar{q}$ vector states, $X_{T, L}^{J=1} \equiv(q \bar{q})_{T, L}^{J=1}$. As the two-gluon-exchange dynamics from QCD for DIS at low $x$ can hardly be doubted, the validity of GVD, or the generalized vector dominance/color-dipole picture (GVD/CDP) has thus been established. Conversely, a violation of the sum rules would imply a failure of the generic two-gluon exchange structure ${ }^{10}$ that can hardly be imagined.

\section{The gluon structure function}

The gluon structure function is quantitatively related to the short-distance behaviour of the color-dipole cross section. Comparing the expression for $\sigma_{\gamma^{*} p}\left(W^{2}, Q^{2}\right)$ in terms of the color-dipole cross section, (2.1), with the one based on the $\gamma^{*} g \rightarrow \gamma^{*} g$ interaction and the gluon-structure function for $\vec{r}_{\perp}^{2} \rightarrow 0$, one finds 21, 22,

$$
\sigma_{(q \bar{q}) p}\left(\vec{r}_{\perp}^{2}, W^{2}\right)=\vec{r}_{\perp}^{2} \frac{\pi^{2}}{3} \alpha_{s}\left(Q^{2}\right) x g\left(x, Q^{2}\right) .
$$

According to (2.6) to (2.8), we have

$$
\sigma_{(q \bar{q}) p}\left(\vec{r}_{\perp}^{2}, W^{2}\right)=\vec{r}_{\perp}^{2} \frac{\pi}{4} \int d \vec{l}_{\perp}^{2} \vec{l}_{\perp}^{2} \tilde{\sigma}_{(q \bar{q}) p}\left(\vec{l}_{\perp}^{2}, W^{2}\right)
$$

Equating (4.2) with (4.1), we find

$$
\alpha_{s}\left(Q^{2}\right) x g\left(x, Q^{2}\right)=\frac{3}{4 \pi} \int d \vec{l}_{\perp}^{2} \vec{l}_{\perp}^{2} \tilde{\sigma}_{(q \bar{q}) p}\left(\vec{l}_{\perp}^{2}, W^{2}\right) .
$$

Alternatively, the gluon structure function may be represented as an integral over $\overrightarrow{l_{\perp}^{\prime 2}}$

$$
\alpha_{s}\left(Q^{2}\right) x g\left(x, Q^{2}\right)=\frac{1}{8 \pi} \int d \vec{l}_{\perp}^{\prime 2} \vec{l}_{\perp}^{\prime 2} \bar{\sigma}_{(q \bar{q})_{L}^{J=1}}\left(\vec{l}_{\perp}^{\prime 2}, W^{2}\right) .
$$

Relation (4.4) becomes identical to (4.3) upon substitution of $\bar{\sigma}_{(q \bar{q})_{L}^{J=1}}$ from (2.20). Relation (4.4) says that the gluon structure function is related to the interaction

\footnotetext{
10 "Generic" insofar, as the derivation is independent of the detailed specification of the lower vertices in fig. 1.
} 
of longitudinally polarized $q \bar{q}$ vector states, $(q \bar{q})_{L}^{J=1} p \rightarrow(q \bar{q})_{L}^{J=1} p$. Combining (4.4) with the short-distance expansion of (2.22),

$$
\sigma_{(q \bar{q})_{L}^{J=1} p}\left(\vec{r}_{\perp}^{\prime}, W^{2}\right)=\vec{r}_{\perp}^{\prime 2} \frac{\pi}{4} \int d \vec{l}_{\perp}^{\prime 2} \vec{l}_{\perp}^{\prime 2} \bar{\sigma}_{(q \bar{q})_{L}^{J=1} p}\left(\vec{l}_{\perp}^{\prime 2}, W^{2}\right)
$$

we find

$$
\sigma_{(q \bar{q})_{L}^{J=1} p}\left(\vec{r}_{\perp}^{\prime 2}, W^{2}\right)=\vec{r}_{\perp}^{\prime 2} \cdot 2 \pi^{2} \alpha_{s}\left(Q^{2}\right) x g\left(x, Q^{2}\right) .
$$

Relation (4.6) is the analogue of (4.1), if the representation (2.21) is used for $\sigma_{\gamma_{T, L}^{*} p}\left(W^{2}, Q^{2}\right)$. Relations (4.4) and (4.6) explicitly say that the gluon-structure function is determined by the dipole cross section for longitudinally polarized $q \bar{q}$ vector states. Contributions to the dipole cross section describing inelastic diffraction, $\gamma_{T, L}^{*} p \rightarrow(q \bar{q})_{T, L}^{J \neq 1} p$, are irrelevant as far as the determination of the gluon structure function is concerned.

Finally, we may introduce the unintegrated gluon structure functions

$$
\alpha_{s}\left(Q^{2}\right) \tilde{\mathcal{F}}\left(x, \vec{l}_{\perp}^{2}\right)=\frac{3}{4 \pi} \vec{l}_{\perp}^{2} \tilde{\sigma}_{(q \bar{q}) p}\left(\vec{l}_{\perp}^{2}, W^{2}\right)
$$

as well as, in terms of $\bar{\sigma}_{(q \bar{q})_{L}^{J=1} p}$,

$$
\alpha_{s}\left(Q^{2}\right) \overline{\mathcal{F}}\left(x, \vec{l}_{\perp}^{\prime 2}\right)=\frac{1}{8 \pi} \vec{l}_{\perp}^{\prime 2} \bar{\sigma}_{(q \bar{q})_{L}^{J=1} p}\left(\vec{l}_{\perp}^{\prime 2}, W^{2}\right) .
$$

The gluon structure function is then given by

$$
\alpha_{s}\left(Q^{2}\right) x g\left(x, Q^{2}\right)=\int d \vec{l}_{\perp}^{2} \alpha_{s}\left(Q^{2}\right) \tilde{\mathcal{F}}\left(x, \vec{l}_{\perp}^{2}\right)
$$

and

$$
\alpha_{s}\left(Q^{2}\right) x g\left(x, Q^{2}\right)=\int d \vec{l}_{\perp}^{\prime 2} \alpha_{s}\left(Q^{2}\right) \overline{\mathcal{F}}\left(x, \vec{l}_{\perp}^{\prime 2}\right)
$$

\section{The ansatz for the color-dipole cross section in the GVD/CDP}

In this section, we briefly remind the reader of the ansatz in the GVD/CDP that fits the experimental data for $\sigma_{\gamma_{T, L}^{*} p}\left(W^{2}, Q^{2}\right)$. It was used in our previous derivation [4] of the sum rules from section 3 . 
We go back to the Fourier representation of the color-dipole cross section in (2.17) in terms of the variable $\vec{l}_{\perp}^{\prime}$ and to the cross sections for $(q \bar{q})_{T, L}^{J=1}$ vector states in (2.23) and (2.24). In particular, let us assume the product

$$
z(1-z) \tilde{\sigma}_{(q \bar{q}) p}\left(\vec{l}_{\perp}^{\prime 2} z(1-z), W^{2}\right)=f\left(\vec{l}_{\perp}^{\prime 2}, W^{2}\right)
$$

to be independent of the variable $z$. In this case, the integrations over $d z$ in (2.23) and (2.24) can be carried out, and we have

$$
\bar{\sigma}_{(q \bar{q})_{T}^{J=1}}\left(\vec{l}_{\perp}^{\prime 2}, W^{2}\right)=\bar{\sigma}_{(q \bar{q})_{L}^{J=1}}\left(\vec{l}_{\perp}^{\prime 2}, W^{2}\right)=f\left(\vec{l}_{\perp}^{\prime 2}, W^{2}\right)
$$

Substitution of a suitable function $f\left(\vec{l}_{\perp}^{\prime 2}, W^{2}\right)$ into (2.22) and (2.21), as well as (3.23) and (3.24), yields predictions for $\sigma_{\gamma_{T, L}^{*} p}\left(W^{2}, Q^{2}\right)$ and for the diffractive production cross section for $\gamma_{T, L}^{*} p \rightarrow X_{T, L}^{J=1} p$ in the forward direction.

Our ansatz [5] in the GVD/CDP

$$
\begin{aligned}
& z(1-z) \tilde{\sigma}\left(\vec{l}_{\perp}^{2}, W^{2}\right)=z(1-z) \frac{\sigma^{(\infty)}}{\pi} \delta\left(\vec{l}_{\perp}^{2}-z(1-z) \Lambda^{2}\left(W^{2}\right)\right) \\
& =\frac{\sigma^{(\infty)}}{\pi} \delta\left(\vec{l}_{\perp}^{\prime 2}-\Lambda^{2}\left(W^{2}\right)\right)
\end{aligned}
$$

has precisely the form (5.1), and according to (5.2), it amounts to

$$
\bar{\sigma}_{(q \bar{q})}^{J=1}\left(\vec{l}_{\perp}^{\prime 2}, W^{2}\right)=\bar{\sigma}_{(q \bar{q})_{L}^{J=1}}\left(\vec{l}_{\perp}^{\prime 2}, W^{2}\right)=\sigma^{(\infty)} \cdot \frac{1}{\pi} \delta\left(\vec{l}_{\perp}^{\prime 2}-\Lambda^{2}\left(W^{2}\right)\right) .
$$

With respect to transverse position space, from (2.6) or (2.22), we find

$$
\sigma_{(q \bar{q})_{T}^{J=1} p}\left(\vec{r}_{\perp}^{\prime}, W^{2}\right)=\sigma_{(q \bar{q})_{L}^{J=1} p}\left(\vec{r}_{\perp}^{\prime}, W^{2}\right)=\sigma^{(\infty)}\left(1-J_{0}\left(r_{\perp}^{\prime} \cdot \Lambda\left(W^{2}\right)\right) .\right.
$$

As to the meaning of the ansatz (5.3) to (5.5) , it is worth noting that it is nothing else but an approximation of the (unknown) distribution in the transverse gluon momentum (multplied by $1 / z(1-z)), \vec{l}_{\perp}^{\prime 2}$, by a $\delta$-function that defines the effective value of $\vec{l}_{\perp}^{\prime 2}$ via

$$
\left\langle\vec{l}_{\perp}^{\prime 2}\right\rangle_{W^{2}}=\frac{\int d \vec{l}_{\perp}^{\prime 2} \vec{l}_{\perp}^{\prime 2} \bar{\sigma}_{\left(q \bar{q} J_{L}^{J=1}\right.}\left(\vec{l}_{\perp}^{\prime 2}, W^{2}\right)}{\int d \vec{l}_{\perp}^{2} \bar{\sigma}_{(q \bar{q})_{L}^{J=1}}\left(\vec{l}_{\perp}^{\prime 2}, W^{2}\right)}=\Lambda^{2}\left(W^{2}\right)
$$


The (expected) rise of the effective value of the transverse momentum of the gluons is fitted to the experimental data. In (5.3) and (5.5),$\Lambda^{2}\left(W^{2}\right)=\Lambda^{2}\left(\frac{Q^{2}}{x}\right)$ is a slowly increasing function of $W^{2}$ parameterized by a power law or a logarithm,

$$
\Lambda^{2}\left(W^{2}\right)=\left\{\begin{array}{c}
B\left(\frac{W^{2}}{W_{0}^{2}}+1\right)^{C_{2}}, \\
C_{1}^{\prime} \ln \left(\frac{W^{2}}{W_{0}^{\prime 2}}+C_{2}^{\prime}\right),
\end{array}\right.
$$

where $B, C_{2}, W_{0}^{2}$ and $C_{1}^{\prime}, C_{2}^{\prime}, W_{0}^{\prime 2}$ are fit parameters and $\sigma^{(\infty)}$ is a cross section of typical hadronic size [5]. According to [5], we have $B=2.24 \pm 0.43 \mathrm{GeV}^{2}$, $W_{0}^{2}=1081 \pm 124 \mathrm{GeV}^{2}$ and $C_{2}=0.27 \pm 0.01$.

We finally give the gluon structure function for the ansatz (5.4). It is given by

$$
\alpha_{s}\left(Q^{2}\right) x g\left(x, Q^{2}\right)=\frac{1}{8 \pi^{2}} \sigma^{(\infty)} \Lambda^{2}\left(W^{2}\right) .
$$

This is easily verified by substituting (5.4) into (4.4).

The ansatz (5.3) with its special dependence on $z(1-z)$, according to (5.4), is recognized as a specification of the elastic scattering of $q \bar{q}$ vector states. This is all that is needed to evaluate [4] the total cross sections (3.13) and (3.14) in momentum space, or (2.21) in transverse position space. Substitution of (5.4) into (3.23) and (3.24) yields [4] the cross sections for diffractive production of $q \bar{q}$ vector states.

\section{Duality, gluon structure function and satura- tion}

It is worth emphasizing that the alternative approaches to a theoretical description of DIS at low $x$ in terms of the color-dipole cross section and in terms of the gluon structure function are to be considered as being dual to each other rather than excluding each other. Both descriptions rely on the two-gluon-exchange dynamical mechanism evaluated at low $x$. While the GVD/CDP interprets the two-gluon-exchange dynamics at low $x$ in terms of a $\gamma^{*}(q \bar{q})$ transition with subse-

quent $(q \bar{q})^{J=1} p \rightarrow(q \bar{q})^{J=1} p$ scattering, the notion of the gluon-structure function relies on $\gamma^{*} g \rightarrow \gamma^{*} g$ scattering. The duality of the two pictures (in a restricted kinematical domain) becomes manifest in relations (4.3) and (4.4) that explicitly 
express the gluon structure function in terms of the (momentum-space expression for the) color-dipole cross section.

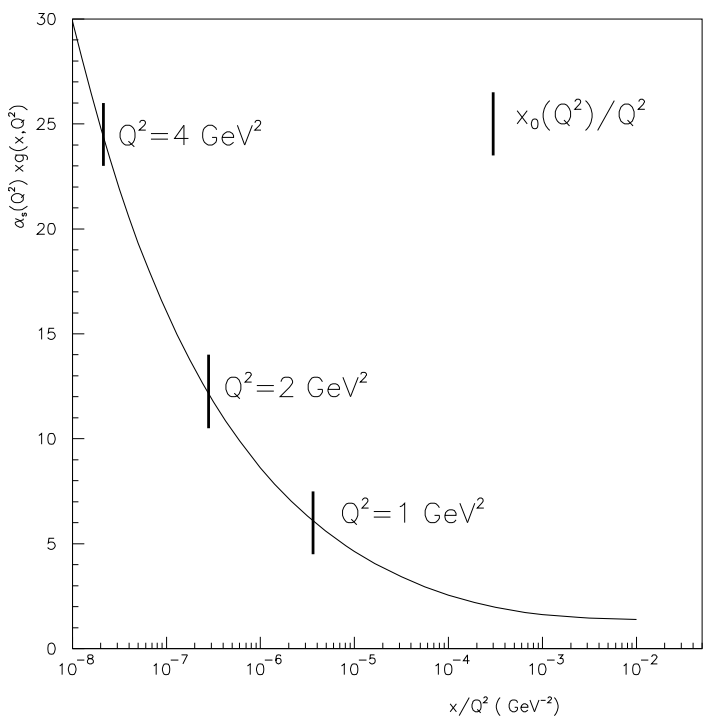

Figure 3: The GVD/CDP prediction of the gluon structure function, $\alpha_{s}\left(Q^{2}\right) x g\left(x, Q^{2}\right)$, as a function of a single variable $x / Q^{2}$. For $x \lesssim x_{0}\left(Q^{2}\right)$, as indicated, the conventional interpretation of the gluon structure function breaks down and "saturation" sets in.

In fig.3, we show the gluon structure function (5.8) of the GVD/CDP. It has the remarkable property of depending only on the energy $W$,

$$
\begin{aligned}
\alpha_{s}\left(Q^{2}\right) x g\left(x, Q^{2}\right) & =\frac{1}{8 \pi^{2}} \sigma^{(\infty)} \Lambda^{2}\left(W^{2}\right) \\
& =\frac{1}{8 \pi^{2}} \sigma^{(\infty)} \Lambda^{2}\left(\frac{Q^{2}}{x}\right)
\end{aligned}
$$

and accordingly, we plot it against

$$
\frac{1}{W^{2}}=\frac{x}{Q^{2}}
$$

We urge experimentalists to plot the gluon structure function ( multiplied by $\left.\alpha_{s}\left(Q^{2}\right)\right)$ they extract from the measured cross sections against $x / Q^{2}$, in order to verify the scaling behavior ${ }^{11}$ of fig.3. In fig.3, we also show selected values for the

\footnotetext{
${ }^{11}$ Note that the scaling is to be considered as a LO QCD result that is best fulfilled for $x \ll 0.1$.
} 
quantity $x_{0}\left(Q^{2}\right)$. The quantity $x_{0}\left(Q^{2}\right)$ defines the kinematical boundary of the region of $x \lesssim x_{0}\left(Q^{2}\right)$ where saturation in the sense of [5]

$$
\lim _{\substack{W^{2} \rightarrow \infty \\ Q^{2} \rightarrow \text { fixed }}} \frac{\sigma_{\gamma^{*} p}\left(W^{2}, Q^{2}\right)}{\sigma_{\gamma p}\left(W^{2}\right)}=1
$$

sets in. Saturation (6.3) sets in for $\eta$ sufficiently small,

$$
\eta \lesssim \eta_{0} \ll 1
$$

where $\eta$ is the scaling variable,

$$
\eta\left(W^{2}, Q^{2}\right)=\frac{Q^{2}+m_{0}^{2}}{\Lambda^{2}\left(W^{2}\right)}
$$

that determines the behavior of the total photoabsorption cross section [5]

$$
\sigma_{\gamma^{*} p}\left(\eta\left(W^{2}, Q^{2}\right)\right)=\frac{2 \alpha}{3 \pi} \sigma^{(\infty)} \begin{cases}\log (1 / \eta), & \text { for } \eta \ll 1, \\ \frac{1}{2 \eta}, & \text { for } \eta \gg 1,\end{cases}
$$

and $\Lambda^{2}\left(W^{2}\right)$ is given by (5.7). With (5.7), the condition on $\eta$ in (6.4) is converted into

$$
\begin{aligned}
x \lesssim x_{0}\left(Q^{2}\right) & =\frac{Q^{2}}{W_{0}^{2}} \frac{1}{\left(\left(\frac{Q^{2}}{B \eta_{0}}\right)^{1 / C_{2}}-1\right)} \\
& \cong \frac{\left(B \eta_{0}\right)^{1 / C_{2}}}{W_{0}^{2}\left(Q^{2}\right)^{1 / C_{2}-1}}
\end{aligned}
$$

The bound $x_{0}\left(Q^{2}\right)$ falls strongly with increasing $Q^{2}$, since $x_{0}\left(Q^{2}\right) \cong 1 / Q^{4}$. The numerical values of $x_{0}\left(Q^{2}\right) / Q^{2}$ in fig.3 are based on $\eta_{0}=0.1$.

With respect to the interpretation of the transition to saturation, it is useful ${ }^{12}$ to substitute the scaling variable (6.5) into the cross section (6.6) and replace $\Lambda^{2}\left(W^{2}\right)$ by the gluon structure function (5.7). We obtain,

$$
\sigma_{\gamma^{*} p}\left(\eta\left(\frac{Q^{2}}{x}, Q^{2}\right)\right)=\frac{2 \alpha}{3 \pi} \sigma^{(\infty)} \begin{cases}\log \frac{8 \pi^{2} \alpha_{s}\left(Q^{2}\right) x g\left(x, Q^{2}\right)}{\sigma^{(\infty)}\left(Q^{2}+m_{0}^{2}\right)}, & \eta \ll 1, \\ \frac{8 \pi^{2} \alpha_{s}\left(Q^{2}\right) x g\left(x, Q^{2}\right)}{2 \sigma^{(\infty)} Q^{2}}, & \eta \gg 1 .\end{cases}
$$

According to (6.8), the transition to the saturation region (obviously) does not imply that the gluon-structure function ceases to increase with decreasing $x$.

\footnotetext{
${ }^{12}$ It is useful for the interpretation of saturation, even though the simple scaling behavior in $\eta$ becomes a hidden one.
} 
The onset of saturation depends on $Q^{2}$ via $x_{0}\left(Q^{2}\right)$, and it simply means that the approximation of $\eta \gg 1$ in (6.6) and (6.8) breaks down, and the logarithmic (soft) behavior sets in. Note that the region of $\eta \gg 1$ is the one where the logarithmic derivative of the structure function $F_{2}=\left(Q^{2} / 4 \pi^{2} \alpha\right) \sigma_{\gamma^{*} p}\left(W^{2}, Q^{2}\right)$ yields the gluon structure function [5] as a result of the evolution equations.

To summarize, saturation does not mean that the gluon structure function ceases to rise. The gluon structure function rises indefinitely for $1 / W^{2}=x / Q^{2} \rightarrow$ 0 . At any fixed $Q^{2}$, however, for $x \lesssim x_{0}\left(Q^{2}\right)$, the conventional connection between $F_{2}$ and the gluon density breaks down, and saturation in the sense of (6.3) sets in. Alternatively, for any fixed $x$, however small, the conventional evolution takes place provided $Q^{2}$ is sufficiently large.

\section{Conclusion}

We end with a brief summary:

i) Quantum chromodynamics, in particular the generic two-gluon-exchange, valid at low $x$, implies a representation of the total photoabsorption cross section as a sum over the mass spectra of diffractive production of $(q \bar{q})$ vector states. In this sense, the generalized vector dominance picture is a consequence of QCD. The GVD/CDP may thus be considered established insofar as its violation would falsify the underlying generic two-gluon-exchange structure - an assumption hardly questionable from all we know about quark and antiquark interactions in QCD.

ii) The kinematic domain of the GVD/CDP, $x \lesssim 0.1$ and $Q^{2}$ arbitrary, includ$\operatorname{ing} Q^{2}=0$, allows one to estimate the kinematic domain where the GVD/CDP and the description in terms of the gluon structure function are dual to each other. Apart from the usual restriction of $Q^{2} \gtrsim Q_{0}^{2}>0$, we find that the duality domain is bounded by $x \gtrsim x_{0}\left(Q^{2}\right)$, where $x_{0}\left(Q^{2}\right)$ is exceedingly small and decreases strongly with increasing $Q^{2}$. For $x \lesssim x_{0}\left(Q^{2}\right)$ saturation sets in. 


\section{References}

[1] F.E. Low, Phys. Rev. D12, 163 (1975);

S. Nussinov, Phys. Rev. Lett. 34, 1286 (1975); Phys. Rev. D14, 246 (1976);

J. Gunion, D. Soper, Phys. Rev. D15, 2617 (1977).

[2] N.N. Nikolaev, B.G.Zakharov, Z. Phys. C49, 607 (1991); Z. Phys. C53, 331 (1992); Soviet Phys. JETP 78, 598 (1994).

[3] J.D. Bjorken, J. Kogut, D.E. Soper, Phys. Rev. D3, 1382 (1971).

[4] M. Kuroda, D. Schildknecht, Phys. Rev. D66, 094005, (2002);

D, Schildknecht and H. Spiesberger, Acta Phys. Pol. B29, 1261 (1998);

M. Kuroda, D. Schildknecht, B. Surrow, M. Tentyukov, Proc. DIS 2002, Acta Phys. Pol. B33, 3431 (2002);

D. Schildknecht, Proc. QCD 2002, ed. by S. Narison, Montpellier, France, hep-ph0209369

[5] D. Schildknecht, at "Diffraction2000", Cetraro, Italy, September 2-7, 2000, Nucl. Phys. B Proc. Suppl. B99, 121 (2001);

D. Schildknecht, B. Surrow, M. Tentyukov, Phys. Lett. B499, 116 (2001); Mod. Phys. Lett. A, Vol. 16, 1829 (2001);

G. Cvetic, D. Schildknecht, B. Surrow, M. Tentyukov, Eur. Phys. J. C20, 77 (2001);

D. Schildknecht and M. Tentyukov, hep-ph/0203028,

B. Surrow, Proc. Int. Europhysics Conf. on High Energy Physics, Budapest, Hungary 2001.

[6] J.J. Sakurai, D. Schildknecht, Phys. Lett. 40B, 121 (1972);

B. Gorczyca, D. Schildknecht, Phys. Lett. 47B, 71 (1973).

[7] H. Fraas, B.J. Read, D. Schildknecht, Nucl. Phys. B86, 346 (1975);

R. Devenish, D. Schildknecht, Phys. Rev. D19, 93 (1976).

[8] A. Donnachie and G. Shaw, in Electromagnetic Interaction of Hadrons, (Plenum Press, N.Y., 1978), vol.2, p.169. 
[9] G. Cvetic, D. Schildknecht, A. Shoshi, Eur. Phys. J. C13, 301 (2000); Acta Phys. Pol. B30, 3265 (1999).

[10] L. Frankfurt, V. Guzey, M. Strikman, Phys. Rev. D58, 094039 (1998).

[11] The ZEUS Collaboration, J. Breitweg et al., Eur. Phys. J. C6, 43 (1999); The H1 Collaboration, C. Adloff et al., Z. Phys. C76, 613 (1997).

[12] J. Forshaw, G. Kerley, G. Shaw, Phys. Rev. D60, 074012 (1999); Nucl. Phys. A675, 80c (2000), hep-ph/9910251.

[13] K. Golec-Biernat, M. Wüsthoff, Phys. Rev. D59, 014017 (1999); Phys. Rev. D60, 114023 (1999);

A.M. Stasto, K. Golec-Biernat, J. Kwiecinski, Phys. Rev. Lett. 86, 596 (2001).

[14] J. Bartels, K. Golec-Biernat, H. Kowalski, hep-ph/0203258.

[15] P. Landshoff, hep-ph/0209364.

[16] A.M. Cooper-Sarkar, R.C.E. Devenish, A.De Roeck, hep-ph/9712301, Int. J. Mod. Phys. A13, 3385 (1998).

[17] V.N. Gribov, Soviet. Phys. JETP30, 709 (1970).

[18] J.J. Sakurai, Phys. Lett., 46B, 207 (1973); Proc. 1973 International School of Subnuclear Physics, Erice, Italy, (ed. A. Zichichi, Academic Press, N.Y.), p.219.

[19] E.M. Levin, A.D. Martin, M.G. Ryskin and T. Teubner, Z. Phys. C74, 671 (1997);

A.D. Martin, M.G. Ryskin and T. Teubner, Phys. Rev. D55, 4329 (1997).

[20] A.Bialas, R. Peschanski and Ch. Royon, Phys. Rev. D57, 6899 (1998).

[21] N.N. Nikolaev, B.G. Zakharov, Phys. Lett. B332, 184 (1994)

[22] L. Frankfurt, A. Radyushkin, M. Strikman, Phys. Rev. D55, 98 (1997). 